\title{
A pore-scale study on the drying kinetics and mechanical behavior of particle aggregates
}

\author{
Pham, T. S. ${ }^{\text {a }}$ Chareyre, B. ${ }^{\text {b,c }}$; Tsotsas, E. ${ }^{\text {a }}$ Kharaghani, A. ${ }^{a^{*}}$ \\ a Department of Thermal Process Engineering, Otto von Guericke University, Magdeburg, Germany \\ b 3SR, University Grenoble-Alpes, Grenoble, France \\ c 3SR, CNRS, Grenoble, France \\ *E-mail of the corresponding author: abdolreza.kharaghani@ovgu.de
}

\begin{abstract}
A discrete thermo-mechanical drying model is developed to investigate the interaction between the porous structure and the drying characteristics of dense particle aggregates. The solid phase consists of polydisperse spherical particles in the micrometer range and the void space is constructed by a complementary network of tetrahedral pores. A modified version of the classical invasion percolation algorithm is set up to describe the preferential evaporation of the confined liquid in the pores. Thus, the evolution of the liquid distribution throughout the complex disordered medium can be simulated. In a one-way coupling scheme, capillary forces caused by both fluid pressure and surface tension are computed over time from the filling state of pores and they are applied as loads on each primary particle in the discrete element method. Based on this robust approach the drying kinetics and the mechanical behavior of several different aggregates with various fractions of small and large particles are simulated and quantified.
\end{abstract}

Keywords: Pore network model; discrete element method; Solid-fluid interaction; capillary force, Convective drying. 


\section{Introduction}

Drying in porous media has been subjected to a wide range of scientific investigation, from soil layers [1] to a porous wick in loop heat pipe [2] or a membrane in a fuel cell [3]. This process consists of various complex events, such as two-phase flow together with phase change, or the interaction between fluid and solid phases, which may cause some undesired mechanical effects. These effects mainly depend upon moisture distribution, which is affected by drying conditions and the porous media skeleton. Substantial efforts have been made to numerically investigate the drying problem at the pore scale. After the pioneered work by Prat [4], many other studies have been conducted to take into account many physical effects, e.g. liquid viscosity [5], gravity [6] or liquid film [7].

In addition, the pore network modeling method has been combined with discrete element method (DEM) to account for the solid phase geometry explicitly [8]. Therefore, local effects on a solid phase (such as cracks or shrinkage) has been described for the case of monosized aggregates having regular [9] or irregular [10] pore structure. Such effects are caused by capillary forces, which depend on the evolution of the liquid-gas interface as well as surface tension during the drying process. The solid phase is defined as a particle aggregate and based on this a pore network is generated using the Voronoi tessellation that can approximate the void phase [11]. However, this Voronoi graph has a shortcoming when applied for a polydisperse aggregate, which is a packing composed of primary particles with different sizes. In this case, regular Delaunay triangulation generates the graph to weighted points, which are the radii of particles [12]. This regular Delaunay triangulation method has been used in a pore assembly approach developed by Bruno et al. [13] in order to study the fluid flow problems in granular porous media. The fluid flow model has been coupled with DEM to calculate the capillary force exerting on each particle of the aggregate. This approach has been further improved and applied for several applications. Based on this approach, Catalano et al. [14] investigated the fluid-solid interaction for the case of oedometer test; Chao Yuan et al. [15] studied the hydromechanical response (shrinkage and swelling) during drainage; Sweijen et al. [16] simulated swelling of super absorbent polymers.

The goal of this work is to develop a versatile discrete model that can simulate the mechanical behavior of particle aggregates during convective drying. In this meso-scale model, the solid phase is explicitly described as an assembly of spherical primary particles. The fluid flow within the void space structure is modeled using the pore assembly method while the geometrical representation and the mechanical behavior of the solid skeleton are simulated by DEM. The coupling between these two parts is achieved by handling the interfacial interaction between the fluid and the solids phase. Impact of the internal structure of the porous media on the drying kinetics, for the case of 3D particle aggregates with various fractions of coarse and fine primary particles, has been presented. 


\section{Materials and Methods}

\subsection{Aggregate generation - solid phase representation}

The solid phase is represented by a packing of particles. This particle aggregate is generated by using YADE [17], which is an open-source software based on the discrete element method. A set of very loose particles with a uniform size distribution is enclosed in a parallelepiped. The resulting packing conforms in a gas-like state with no contacts between particles. Mechanical loading is subsequently applied to confer a solid-like nature to the packing.
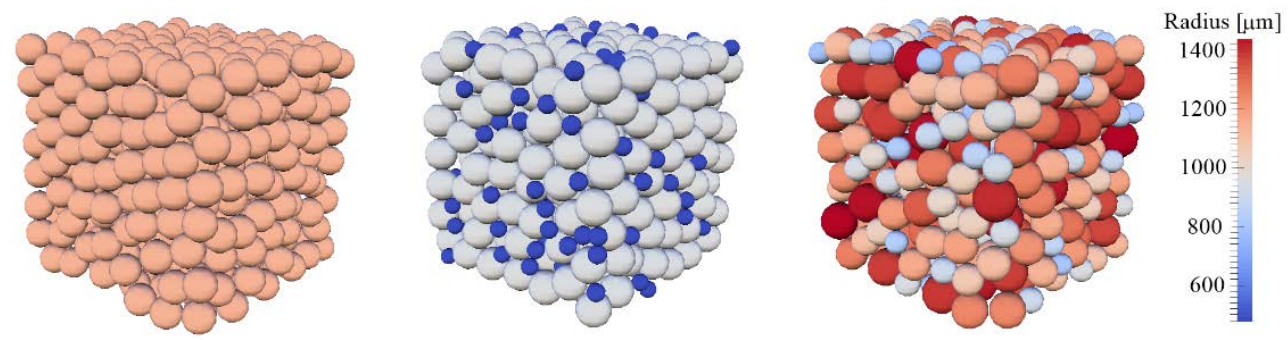

Fig. 1 Representation of monodisperse (left), bidisperse (middle), and polydisperse (right) particle aggregates.

\subsection{Pore network generation - void space representation}

The void space between primary particles is represented by a network of pore bodies and pore throats (see Figs. 2 and 3). This pore network is defined by a weighted Delaunay tessellation of the granular assembly. The pore body is subtracted from the finite volumes of the corresponding tetrahedral cell, which has its vertices locating at centers of associated particles (Fig. 2a), by the part of the solid volume of these associated particles locating within the domain of the tetrahedral cell. Each pair of pore bodies is connected to each other through one common facet (pore throat) between them (Fig. 2b). This pore throat has no volume and serves as the capillary threshold between the two corresponding pore bodies. Therefore, at a given time, the geometry of the packing defines an open network of connected voids.
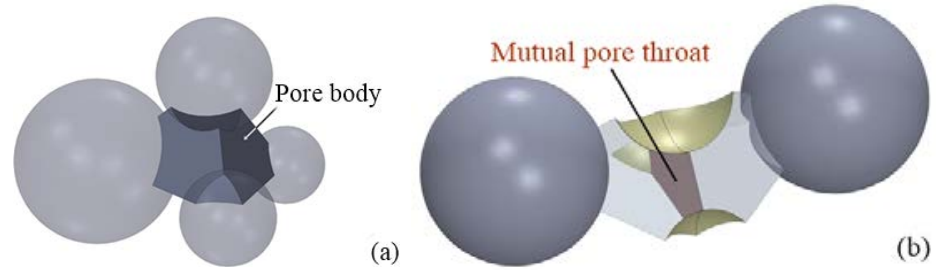

Fig. 2 Representation of a pore body and associated neighboring particles (a) and of two neighboring pore bodies sharing one mutual pore throat (b). 


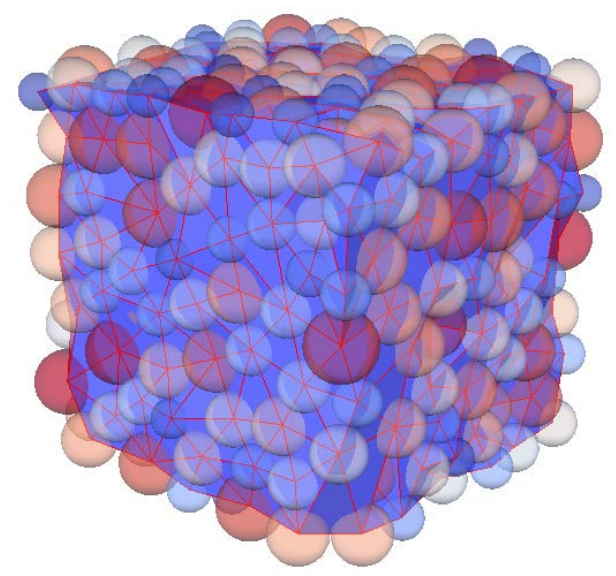

Fig. 3 Pore network triangulated based on the particle aggregate.

\subsection{Drying algorithm}

The isothermal discrete drying algorithm used in this work was based on the work of Metzger et al. [18]. The drying phenomenon is interpreted as an invasion percolation driven by evaporation of liquid into vapor. Vapor diffusion in gas-filled pore bodies is governed by diffusion with Stefan correction. The boundary conditions are applied here for the vapor pressure as saturation value at liquid-gas interface and value of bulk air at top of boundary layer. Besides, the pore size is big enough (more than $100 \mu \mathrm{m}$ ) so that the Kelvin effect, i.e. the reduction of equilibrium vapor pressure over a curved liquid-vapor interfaces may be neglected [18]. The movement of this menisci is not tracked continuously since the time is discretized based on each complete emptying of one single pore in each simulation step. This is due to the assumption that the movement of the liquid-vapor interface, which can be referred to as a Haines jump [19], is much faster than the duration of the evaporation occurring at that meniscus. During each calculation step, vapor flow rates are assumed as constant without accounting for local accumulation of vapor in the gas phase. Additionally, in the gas-filled section of the partially saturated pore bodies, it is assumed that the gas is saturated with vapor (local equilibrium), hence neglecting any vapor resistance in this section. Then, the following mass balances are applied for all gas-filled pore bodies of unknown vapor pressure pv:

$$
\sum_{j=1}^{4} \dot{M}_{v, i}=\sum_{j=1}^{4} A_{i j} \frac{\delta}{L_{i j}} \frac{p \tilde{M}_{v}}{\widetilde{R} T} \ln \left(\frac{p-p_{v, i}}{p-p_{v, j}}\right)=0
$$

Due to the random spatial distribution of the pore size, the liquid phase in the liquid-filled region of the network tends to be gradually separated into numerous isolated liquid clusters [6]. In the absence of viscous and gravity forces, liquid flow within these isolated clusters is totally controlled by capillary pressure, which is the pressure difference at the liquid-gas interface in each pore body locating at the boundary of each liquid cluster. If it is assumed that the gas pressure is the same everywhere within the gas-filled section of the void space, 
then due to capillary pumping effect there is only one pore body in each liquid cluster, whose saturation changes in each calculation step. This pore body is the one having the highest liquid pressure, i.e. lowest capillary pressure, and it will hereafter be referred as entry pore. For every moment of the simulation, each liquid cluster has one entry pore and different entry pores can have different pore volume, pore saturation and evaporation rate. Consequently, the time required to totally invade these entry pores will be different. Therefore, the time step of the drying simulation is determined based on the liquid cluster which has its entry pore empty totally first.

\subsection{Capillary force calculation}

The capillary force exerting on each particle consists of two contributions. The first contribution is induced by the fluid pressure in pore bodies. It is calculated based on the gas and liquid pressure and the surface area of the solid particle being in contact with each corresponding fluids. The second capillary force contribution is the result of interfacial tension acting on the three phase (solid-liquid-gas) contact line. The method used for calculating this capillary force follows the work in [16].

\section{Simulation results}

We consider here a three-dimensional pore network initially saturated with single-component liquid (pure water). In this network the effects of gravity and liquid viscosity are neglected. The water vapor can only elude from the network through the boundary layer locating at the top surface of the sample while a zero-flux condition is applied at other surfaces. It is also assumed that the bulk air is totally dry, i.e. vapor pressure is equal to zero. The temperature of the entire network is uniform at $20{ }^{\circ} \mathrm{C}$. We also neglect the liquid film effect - which can be significant for pores with square or polygonal cross section [21].
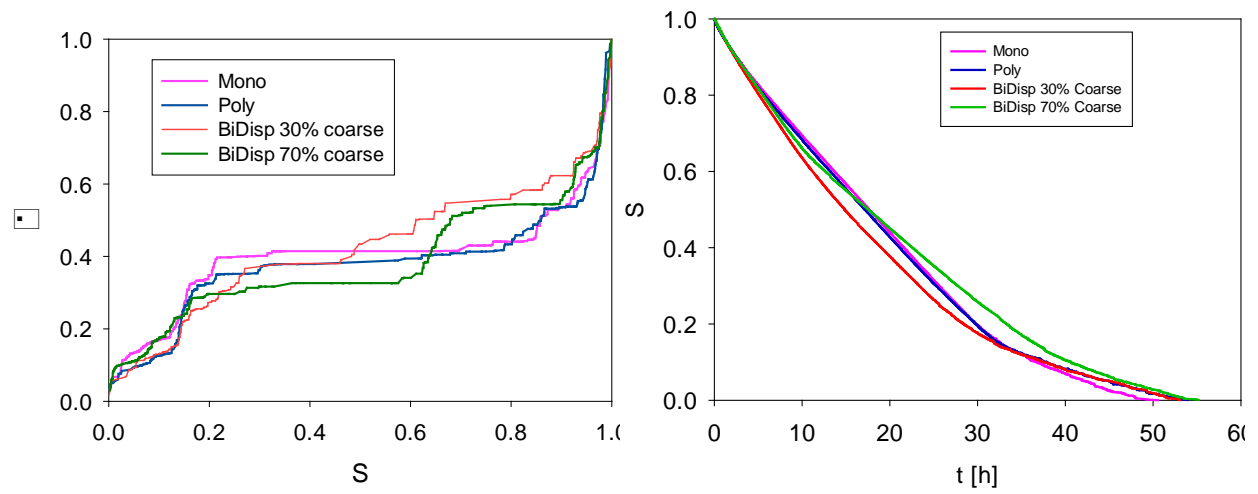

Fig. 4 Normalized drying curves of mono, poly and bidisperse particle aggregates. 
The drying curves obtained for four different particle size distributions, i.e. mono, poly and two bidisperse aggregates with different volume fractions of coarse and fine particles, are presented in Figure 4. These curves show similar drying characteristics: a sharp drop at the beginning followed by a semi-constant evaporation rate period and ended with the falling rate period. These observation is in agreement with previous works [20]. Besides, the total time required to dry the aggregates are also presented. Although these four aggregates have approximately equal porosity and the same physical size of the whole domain, they still have differences in the spatial distribution of the fluid channel within the network. The liquid distribution obtained from the fluid flow calculation in each invasion step is the input for the calculation of capillary force in DEM module. Dynamic simulations are run to visualize the evolution of capillary forces during the drying process of the polydisperse aggregate (Fig. 5). Such capillary forces will subsequently be loaded to primary particles as external loads, and thus the particle motion shall be computed using DEM. We conjecture that different local distributions of fluid can lead to different mechanical responses. Detailed and systematical analysis will be addressed in the near future.
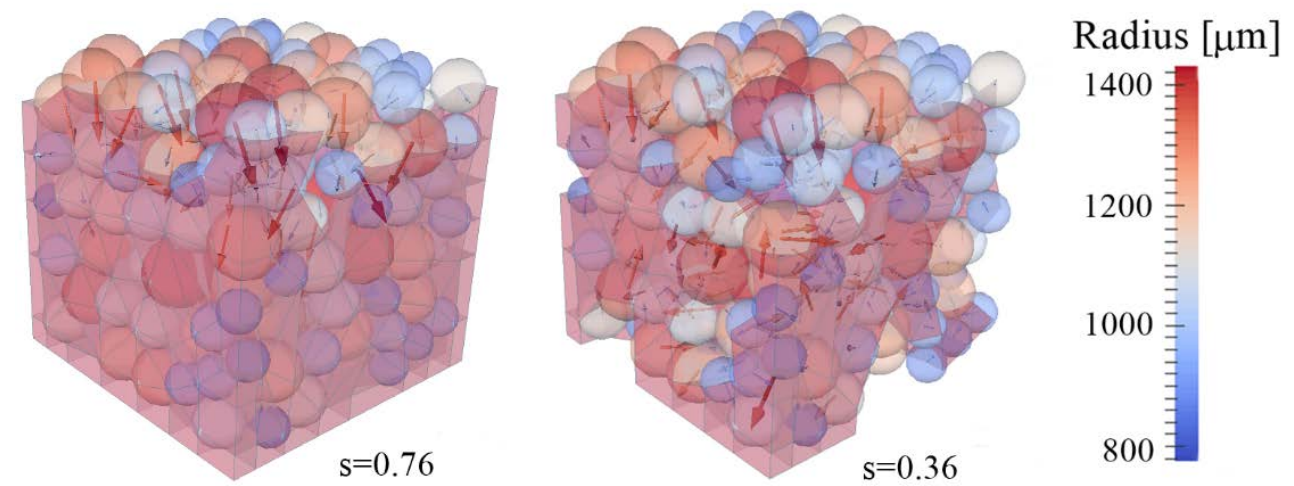

Fig. 5 Evolution of capillary force field (shown by arrows) in a polydisperse particle aggregate during drying.

\section{Conclusion}

A new discrete pore network model has been developed to investigate the fluid flow problem in dense particle aggregates consisting of spherical particles. The proposed approach integrates the pore assembly method with DEM. The aggregate was first generated using DEM based on given micro-scale properties, i.e. porosity, geometrical dimension and particle size distribution. Then, a numerical algorithm is developed to solve the fluid flow problem for the case of convective drying. It was shown that the developed model is capable of capturing the typical characteristic of this classical drying problem, i.e. constant rate period, falling rate period. Moreover, the obtained liquid distribution is then used as an input for calculating the capillary force field exerting on each particle of the aggregate. This is the first 
step to couple the fluid flow model with DEM in order to simulate the thermo-mechanical behavior of polydisperse particle aggregates during drying.

\section{Nomenclature}

$\begin{array}{cll}\dot{M} & \text { Mass flow rate } & \mathrm{kgs}^{-1} \\ \text { A } & \text { Area of cross section } & \mathrm{m}^{2} \\ \mathrm{~L} & \begin{array}{l}\text { Distance between the center of } \\ \text { two adjacent pore bodies }\end{array} & \mathrm{m} \\ \mathrm{p} & \text { Atmospheric pressure } & \mathrm{Pa} \\ \tilde{M} & \text { Molar mass } & \mathrm{kgkmol}^{-1} \\ \tilde{R} & \text { Ideal gas constant } & \mathrm{Jkmol}^{-1} \mathrm{~K}^{-1} \\ \mathrm{~T} & \text { Temperature } & \mathrm{K}\end{array}$

Greek letters

$\begin{array}{lll}\delta & \text { Vapor diffusivity } & \mathrm{m}^{2} \mathrm{~s}^{-1} \\ \gamma & \text { Surface tension } & \mathrm{Nm}^{-1}\end{array}$

Subscripts

V vapor

\section{References}

[1] Or, D.; Lehmann, P.; Shahraeeni, E.; Shokri, N. Advances in Soil Evaporation PhysicsA Review. Vadose Zone Journal 2013, 12(4).

[2] Le, K.H.; Kharaghani, A.; Kirsch, C.; Tsotsas, E. Pore Network Simulations of Heat and Mass Transfer inside an Unsaturated Capillary Porous Wick in the Dry-out Regime. Transport in porous media 2016, 114(3), 623-648.

[3] Straubhaar, B.; Pauchet, J.; Prat, M. Pore network modelling of condensation in gas diffusion layers of proton exchange membrane fuel cells. International Journal of Heat and Mass Transfer 2016, 102, 891-901.

[4] Prat, M. Percolation model of drying under isothermal conditions in porous media. International Journal of Multiphase Flow 1993, 19(4), 691-704.

[5] Nowicki, S.C.; Davis, H.T.; Scriven, L.E. Microscopic determination of transport parameters in drying porous media. Drying Technology 1992, 10(4), 925-946.

[6] Laurindo, J.B.; Prat, M. Numerical and experimental network study of evaporation in capillary porous media. Phase distributions. Chemical Engineering Science 1996, 51(23), 5171-5185. 
[7] Yiotis, A.G.; Boudouvis, A.G.; Stubos, A.K.; Tsimpanogiannis, I.N.; Yortsos, Y.C. Effect of liquid films on the drying of porous media. AIChE Journal 2004, 50(11), 27212737.

[8] Kharaghani, A. Irregular Pore Networks and Mechanical Effects During Drying of Porous Media. PhD thesis: Magdeburg, 2010.

[9] Kharaghani, A.; Metzger, T.; Tsotsas, E. A proposal for discrete modeling of mechanical effects during drying, combining pore networks with DEM. AIChE Journal 2011, 57(4), 872-885.

[10] Kharaghani, A.; Metzger, T.; Tsotsas, E. An irregular pore network model for convective drying and resulting damage of particle aggregates. Chemical Engineering Science 2012, 75, 267-278.

[11] Aurenhammer, F. Voronoi diagrams---a survey of a fundamental geometric data structure. ACM Computing Surveys 1991, 23(3), 345-405.

[12] Edelsbrunner, H.; Shah, N.R. Incremental topological flipping works for regular triangulations. Algorithmica 1996, 15(3), 223-241.

[13] Chareyre, B.; Cortis, A.; Catalano, E.; Barthélemy, E. Pore-Scale Modeling of Viscous Flow and Induced Forces in Dense Sphere Packings. Transport in porous media 2012, 92(2), 473-493.

[14] Catalano, E.; Chareyre, B.; Barthélémy, E. Pore-scale modeling of fluid-particles interaction and emerging poromechanical effects. International Journal for Numerical and Analytical Methods in Geomechanics 2014, 38(1), 51-71.

[15] Yuan, C.; Chareyre, B.; Darve, F. Deformation and stresses upon drainage of an idealized granular material. Acta Geotechnica 2017, 40(3), 405.

[16] Sweijen, T.; Nikooee, E.; Hassanizadeh, S.M.; Chareyre, B. The Effects of Swelling and Porosity Change on Capillarity. Transport in porous media 2016, 113, 207-226.

[17] Smilauer, V.; Catalano, E.; Chareyre, B.; Dorofeenko, S.; Duriez, J.; Dyck, N.; Elias, J.; Er, B.; Eulitz, A.; Gladky, A.; et al. Reference Manual; Zenodo, 2015.

[18] Metzger, T.; Tsotsas, E.; Prat, M. Pore-Network Models: A Powerful Tool to Study Drying at the Pore Level and Understand the Influence of Structure on Drying Kinetics: in "Modern Drying Technology: Computational Tools at Different Scales, Vol. 1. Edited by A.Mujjumdar and E.Tsotsas, 2007.

[19] Armstrong, R.T.; Berg, S. Interfacial velocities and capillary pressure gradients during Haines jumps. Physical review. E, Statistical, nonlinear, and soft matter physics 2013, 88(4), 43010.

[20] Yiotis, A.G.; Tsimpanogiannis, I.N.; Stubos, A.K.; Yortsos, Y.C. Pore-network study of the characteristic periods in the drying of porous materials. Journal of colloid and interface science 2006, 297(2), 738-748. 